\title{
Extreme lightwave electron field emission from a nanotip
}

\author{
Dominique Matte, ${ }^{1,2}$ Nima Chamanara,,${ }^{1,2}$ Lauren Gingras, ${ }^{1,2}$ Laurent P. René de Cotret, ${ }^{1,2,3}$ Tristan L. Britt, ${ }^{1,2,3}$ \\ Bradley J. Siwick, ${ }^{1,2,3}$ and David G. Cooke $\oplus^{1,2, *}$ \\ ${ }^{1}$ Centre for the Physics of Materials, McGill University, Montreal, Quebec, Canada H3A 2T8 \\ ${ }^{2}$ Department of Physics, McGill University, Montreal, Quebec, Canada H3A 2 T8 \\ ${ }^{3}$ Department of Chemistry, McGill University, Montreal, Quebec, Canada H3A OB8
}

(Received 25 June 2020; accepted 7 January 2021; published 11 February 2021)

\begin{abstract}
We report on subcycle terahertz light-field emission of electrons from tungsten nanotips under extreme conditions corresponding to a Keldysh parameter $\gamma_{\mathrm{K}} \approx 10^{-4}$. Local peak THz fields up to $40 \mathrm{GV} / \mathrm{m}$ are achieved at the apex of an illuminated nanotip, causing subcycle cold-field electron emission and acceleration in the quasistatic field. By simultaneous measurement of the electron bunch charge and energy distribution, we perform a quantitative test of quasistatic Fowler-Nordheim tunneling theory under field conditions that completely suppress the tunnel barrier. Very high bunch charges of $\sim 10^{6}$ electrons/pulse are observed, reaching maximum energies of $3.5 \mathrm{keV}$ after acceleration in the local field. The energy distribution and emission current show good agreement with Fowler-Nordheim theory even in this extreme field regime. Application of this theory under such extreme THz field conditions predicts a single-shot electron energy distribution with a spectral purity reaching $10^{-4}$. THz field-induced reshaping and sharpening of the nanotip is observed, reducing the tip radius from 120 to $35 \mathrm{~nm}$ over roughly $10^{9} \mathrm{THz}$ shots. These results indicate THz-driven nanotips in the extreme field limit are promising electron sources for ultrafast electron diffraction and microscopy.
\end{abstract}

DOI: 10.1103/PhysRevResearch.3.013137

\section{INTRODUCTION}

Light-matter interactions enter the extreme limit when the energy scale of the field interaction meets or exceeds a characteristic excitation energy of the system [1,2]. In electron photoemission from a metal surface, the relevant energy scale is the work function, typically a few eV. The electron surface potential is transiently tilted in a light field applied normal to the surface, permitting electrons to tunnel to the vacuum at a rate that depends exponentially on the fielddependent width of the barrier [3,4]. The ratio of the tunneling time to the period of the light field defines the Keldysh parameter, $\gamma_{\mathrm{K}}=\tau_{\text {tun }} / T$, and for $\gamma_{\mathrm{K}} \ll 1$ the field interaction is quasistatic [5-7]. For near-infrared to visible light, the light intensities required to reach $\gamma_{\mathrm{K}}=1$ are on the order of $10^{13} \mathrm{~W} / \mathrm{cm}^{2}$, exceeding the damage threshold of metals [8]. Thus, quasistatic light-matter interactions under such extreme conditions have been relatively unexplored in the solid state.

Intense, single-cycle terahertz $(\mathrm{THz})$ pulses illuminating metal nanotips provide a means to explore this extreme lightmatter interaction regime [9-13]. Localized to the tip apex, a broadband local field enhancement occurs via a lightning rod effect that scales as $\eta \sim \lambda / R$, where $R$ is the nanotip radius

\footnotetext{
*cooke@physics.mcgill.ca

Published by the American Physical Society under the terms of the Creative Commons Attribution 4.0 International license. Further distribution of this work must maintain attribution to the author(s) and the published article's title, journal citation, and DOI.
}

and $\lambda$ is the vacuum wavelength [11]. Intense single-cycle $\mathrm{THz}$ pulses with peak electric fields of $1-10 \mathrm{MV} / \mathrm{m}$ become locally enhanced by $\eta>1000$ to several $\mathrm{GV} / \mathrm{m}$ at the nanotip surface. While dc fields of this magnitude would immediately cause catastrophic damage, breakdown is strongly suppressed at higher frequencies under ac field conditions [14]. Moreover, under pulsed illumination the threshold field for breakdown scales with the pulse duration as $\tau^{-4}$ [15].

These $\mathrm{GV} / \mathrm{m}$ local fields exceed the threshold for subcycle cold-field emission from the metal nanotip [6,11,16,17]. Field-emitted electrons drawn directly from the Fermi level are subsequently accelerated to several $\mathrm{keV}$ in the quasistatic field before leaving the local field enhancement region that decays over length scales comparable to the tip radius. Thus the electrons follow a deterministic path governed by the near instantaneous field under which they are ejected. These coldfield-emitted electrons have potential applications as seeds for $\mathrm{THz}$-based accelerators and sources for time-resolved electron diffraction [18] and time-resolved point-projection electron microscopy [19]. For many applications, particularly those seeking near single-shot operation, electron bunch charges of $>10^{6}$ electrons/pulse are typically required for simple, few atom unit cells [20]. Electron bunch charges up to 100 electrons/pulse have been reported from nanotips driven by midinfrared pulses with $\gamma_{\mathrm{K}} \sim 0.1$ [16]. We note that larger bunch charges were likely achieved, but not quantitatively measured, using low repetition rate, intense THz pulses [11].

In this paper, we demonstrate subcycle $\mathrm{THz}$ field emission of electron pulses from a tungsten nanotip in the extreme field limit corresponding to $\gamma_{\mathrm{K}} \sim 10^{-4}$, deep into the quasistatic regime. Average emission currents $>0.1 \mathrm{nA}$ are measured 
corresponding to bunch charges of $\sim 10^{6}$ electrons/pulse. We simultaneously measure the electron energy distribution, with peak energies up to $3.5 \mathrm{keV}$, comparable to previous work [11]. We test this emission against quasistatic FowlerNordheim theory and find good agreement despite these field conditions being conceptually well beyond the limitations of the model. Finally, a pronounced sharpening of the nanotip is observed under these extreme field conditions, gradually reducing the size of the tip from its initial $120-\mathrm{nm}$ radius to sub-35 nm over an estimated $10^{9}$ shots.

\section{METHODS}

\section{A. Intense THz generation and electron detection}

A schematic of the experiment is shown in Fig. 1(a). Single-cycle $\mathrm{THz}$ pulses are generated by tilted pulse-front optical rectification in a room-temperature, $\mathrm{MgO}: \mathrm{LiNbO}_{3}$ prism pumped by $800-\mathrm{nm}$, 4-mJ laser pulses of $120 \mathrm{fs}$ duration at a $1 \mathrm{kHz}$ repetition rate $[21,22]$. Intense $\mathrm{THz}$ pulses are focused to a near diffraction-limited spot on a tungsten nanotip with linear polarization aligned to the nanotip axis, held within a small vacuum chamber with a base pressure of $\sim 10^{-7}$ Torr. Tips were prepared from a polycrystalline tungsten wire using standard electrochemical etching in a $\mathrm{KOH}$ solution [23]. The nanotip radius was initially $120 \mathrm{~nm}$, as verified by scanning electron microscopy (see Fig. 4). The transmitted $\mathrm{THz}$ pulse is minimally perturbed by the presence of the nanotip and is 1:1 imaged on a $200-\mu \mathrm{m}$-thick, (110)-cut GaP crystal for free-space electro-optic sampling of the $\mathrm{THz}$ field. The most intense $\mathrm{THz}$ wave form experienced by the tip is shown in Fig. 1(b) with its amplitude spectrum shown in the inset. The fields are calibrated assuming a GaP electro-optic coefficient $r_{41}=0.97 \mathrm{pm} / \mathrm{V}$ [24], accounting for the Fresnel loss of the final high-resistivity silicon vacuum chamber window. A pair of wire grid polarizers was used to vary the incident $\mathrm{THz}$ peak field strength without changing its polarization state. The tungsten nanotip can be actuated in all three directions inside the chamber through the $\mathrm{THz}$ focus over a several $\mathrm{mm}$ range by moving the nanotip holder coupled through a baffle.

The electron energy distribution and bunch charge are measured simultaneously via a Faraday cup detector positioned less than $1 \mathrm{~cm}$ away from the tip along the emission axis, with a varying stopping potential $\left(V_{\mathrm{s}}\right)$ applied to a grid placed before the detector. Additionally, an electrical connection is made to the tip allowing a direct measurement of the total current being drawn through the tip. These two currents are measured simultaneously using two Keithley 6517B electrometers.

\section{B. Numerical simulations: Local field enhancement}

Three-dimensional (3D) finite-difference time-domain (FDTD) simulations were performed to model the local $\mathrm{THz}$ fields coupled to tungsten nanotips. The nanotip shape was
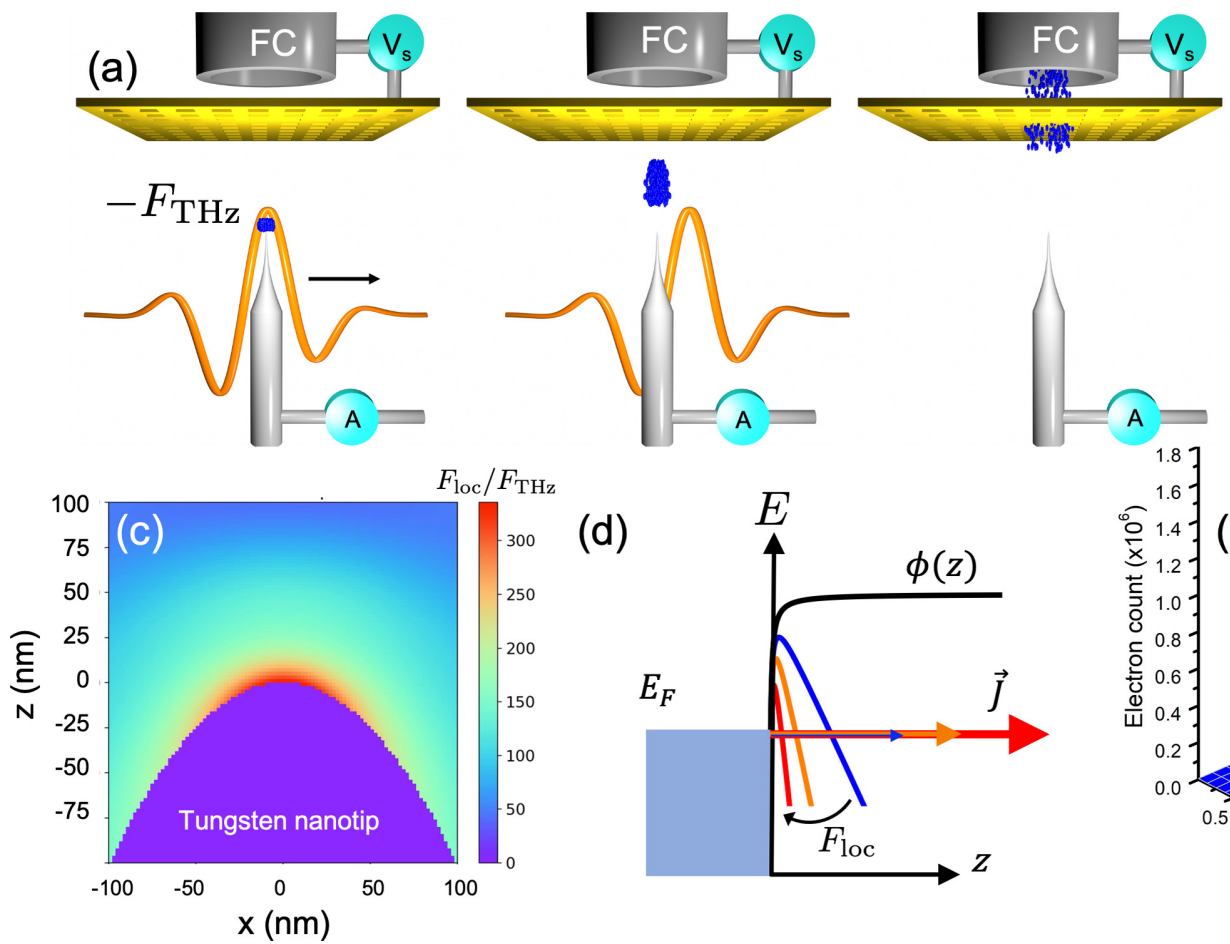

(d)
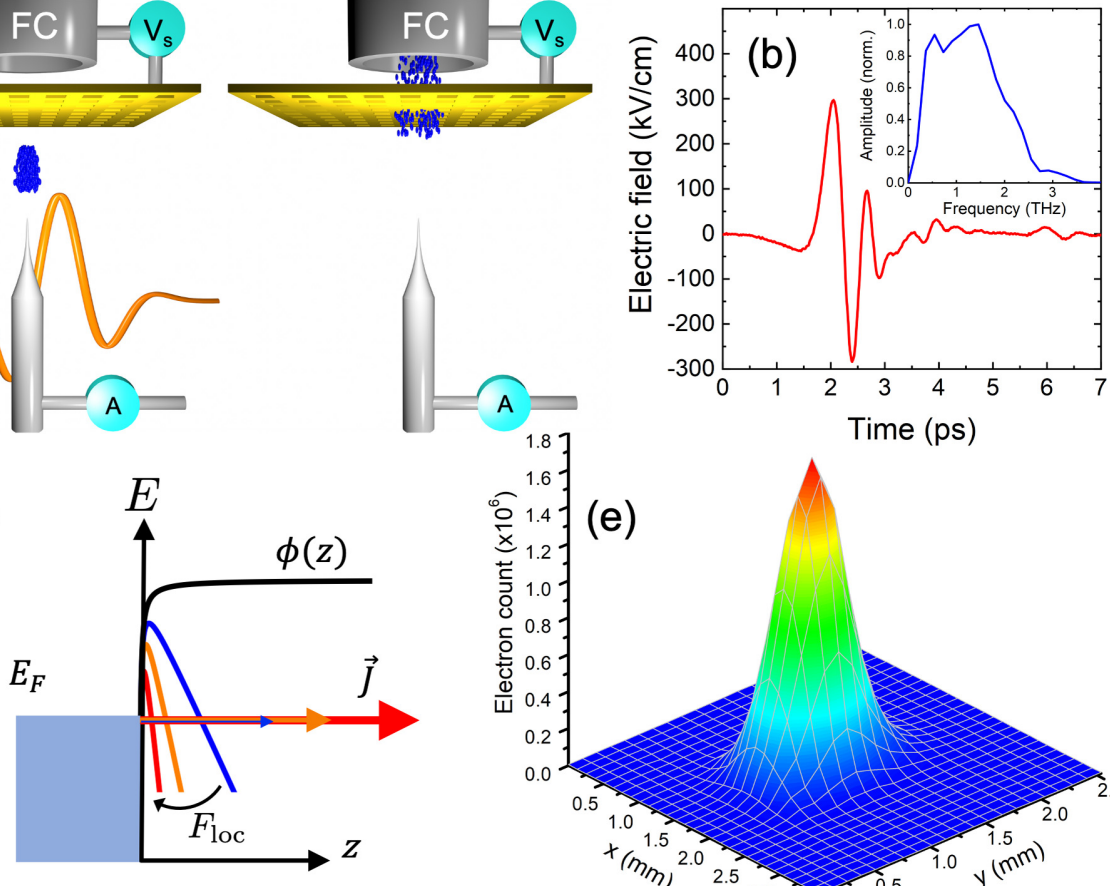

FIG. 1. (a) Schematic of the experiment, whereby a single-cycle $\mathrm{THz}$ pulse $F_{\mathrm{THz}}(t)$ with polarization aligned along the nanotip axis illuminates a metal nanotip and ejects electrons towards a Faraday cup after passing through a grid held at a variable stopping potential $V_{\mathrm{s}}$. (b) Electro-optic sampling of the incident THz pulses within the vacuum chamber illuminate the tip, with a peak $\mathrm{THz}$ field of $298 \mathrm{kV} / \mathrm{cm}$ and Fourier amplitude spectrum shown in the inset. (c) Three-dimensional finite-difference time-domain simulation of the near-field enhancement normal to the tip apex in the vicinity of a 50-nm radius tip. (d) Schematic of the electron potential at the metal-vacuum interface and the field-induced tilting leading to a tunnel current. (e) The spatially resolved electron count per THz pulse as the nanotip is scanned through the fixed $\mathrm{THz}$ focal spot. 
approximated as a prolate spheroid, which allowed the expansion of the fields in spherical coordinates where the Helmholtz equation is separable [25]. The $\mathrm{THz}$ near field is calculated to be of single-cycle character, exhibiting resonances determined mainly by the effective length of the tip and a phase lag (see Fig. 10). This is in agreement with a recent study employing a circuit model for the near-field enhancement [26]. Typical field enhancements of $\sim 350$ are calculated for a 50-nm radius tip, as shown in Fig. 1(c) using the input field given in Fig. 1(b) (see the Appendix for details). While this dipole model qualitatively captures the decay of the local field enhancement away from the tip apex, it ignores the atomic scale structure of the tip and spatial texture in the local field that would be observed in a field ion microscopy image, for example. A more accurate estimation of the local field at the point of emission can be obtained from the measured energy distribution as discussed below.

\section{TERAHERTZ DRIVEN ELECTRON EMISSION}

\section{A. Fowler-Nordheim theory of electron field emission}

Cold-field emission of electrons from metal nanotips under dc and quasi-dc field conditions, depicted in Fig. 1(d), is typically described by Fowler-Nordheim theory [3]. The instantaneous local $\mathrm{THz}$ field $F_{\text {loc }}$ tilts the electron potential given by the Schottky-Nordheim function $V(z)=\phi-$ $F_{\text {loc }} z-\frac{e}{16 \pi \epsilon_{0} z}$ with $\phi=4.5 \mathrm{eV}$ being the work function of the tungsten tip. The critical field required to lower this potential barrier to zero relative to the Fermi energy is given by $F_{\phi}=\frac{4 \pi \epsilon_{0} \phi^{2}}{e}=14 \mathrm{GV} / \mathrm{m}$ for tungsten [27]. The emission current $\vec{J}$ is subsequently calculated via the tunneling probability through this barrier. The zero-temperature Fowler-Nordheim equation for the current density $J$ can then be written as

$$
J(t)=\frac{a F_{\mathrm{loc}}(t)^{2}}{F_{\phi}^{2}} \exp \left[-v b \frac{F_{\phi}}{F_{\mathrm{loc}}(t)}\right],
$$

with $a=\frac{a_{\mathrm{FN}} F_{\phi}^{2}}{\phi} \approx 6.71 \mathrm{GA} / \mathrm{cm}^{2}, \quad b=\frac{b_{\mathrm{FN}} \phi^{3 / 2}}{F_{\phi}} \approx 4.65$, and $a_{\mathrm{FN}}=\frac{e^{3}}{16 \pi^{2} \hbar}$ and $b_{\mathrm{FN}}=\frac{4}{3} \frac{\left(2 m_{\mathrm{e}}\right)^{1 / 2}}{e \hbar}$ are the Fowler-Nordheim constants. The function $v \approx 1-F_{\text {loc }} / F_{\phi}$ accounts for image charges and exponentially suppresses the emission, although it is valid only for $F_{\text {loc }}<F_{\phi}$ [28]. Since the influence of the image charges is limited to very close to the tip apex where local fields are the strongest, we find all data can be described by setting $v=1$ (see the Appendix for details).

\section{B. Electron bunch charge}

The total electron emission $\left(V_{\mathrm{s}}=0 \mathrm{~V}\right)$ is shown in Fig. 1(e), measured using a direct electrical connection on the nanotip and scanning the tip through the focal plane of the $\mathrm{THz}$ pulse. Electron bunch charges in excess of $10^{6}$ electrons/pulse were measured on peak, more than three orders higher than previously reported [16,29]. The relative $\mathrm{THz} /$ tip-position dependence of the field-emission current (bunch charge) follows an asymmetric Gaussian with a full width at half maximum (FWHM) of 580 and $450 \mu \mathrm{m}$ in the $x$ and $y$ directions, respectively [Fig. 1(e)]. These dimensions are comparable to those of the $\mathrm{THz}$ intensity distribution at the tip position as measured using a microbolometer camera. While Fowler-Nordheim emission predicts a sharpened distribution for $F_{\text {loc }} \ll F_{\phi}$ due to the exponential dependence on the field, in the extreme limit of $F_{\text {loc }} \gg F_{\phi}$ the exponential term saturates and the electron distribution is governed by $F_{\text {loc }}^{2}$, i.e., the intensity distribution. Thus both the large electron count rate and the Gaussian spatial distribution indicate that we are operating in the extreme limit of field emission.

\section{Electron emission spectra}

The normalized electron current from the Faraday cup is shown in Fig. 2(a) for varying peak incident $\mathrm{THz}$ field strength. The current shows a marked reduction at low potentials $(<500 \mathrm{eV})$ and a high-energy cutoff at several $\mathrm{keV}$. The numerical derivative of this spectrum gives the energy distribution, whose low- and high-energy regions are shown in Fig. 2(b). The low-energy distribution shows a monotonically decreasing distribution to a cutoff of approximately $400 \mathrm{eV}$, while the high-energy distribution is sharply peaked at energies up to $3.5 \mathrm{keV}$. The origin of the low-energy distribution is puzzling. These low-energy electrons are significantly deflected by the presence of a small permanent magnet resulting in a strong reduction in the signal (see Fig. 9 in the Appendix). The influence of the magnet gradually diminishes at higher electron energies. This confirms that these low-energy electrons originate at the nanotip. Given the significant portion of electrons present in the low-energy part of the distribution, these electrons must be field emitted during the peak of the $\mathrm{THz}$ field. Electrons emitted during this time would usually be accelerated ballistically to form the high-energy peaked distribution. Evidently, a significant portion of electrons are not immediately swept out of the tip region but instead experiences a lower accelerating THz field. Such a delayed photoemission channel has recently been demonstrated using few-cycle optical pulses, where rescattered electrons are driven back to the nanotip surface by subsequent field cycles [30]. Inelastic scattering within the metal can result in a delay of the electron emission by tens of fs. While this cannot explain our results, it points to the role of inelastic scattering during the emission process.

A possible mechanism for such a significant low-energy population is through interactions with surface contaminants. Initially emitted electrons can scatter within contaminant layers [31], subsequently becoming trapped. They can eventually escape and accelerate in a subsequent cycle of the $\mathrm{THz}$ field. Numerical simulations of $\mathrm{THz}$ resonances excited in the nanotip show that a radially polarized, dispersionless Sommerfeld wave is launched on the nanotip [32,33]. This wave reflects at the tip boundaries resulting in a multicycle field at a resonant frequency of $\omega_{0}=(2 \pi) c / 2 L$ where $L \sim 5 \mathrm{~mm}$ is the tip length. As the local field enhancement favors low frequencies (numerically $\eta \propto \omega^{-1.4}$, see Fig. 10 in the Appendix), as does the ponderomotive energy $U_{p}=$ $e^{2} F_{\text {loc }}^{2} / 4 m \omega_{0}^{2}$, electrons can reach $400 \mathrm{eV}$ in this multicycle field with $F_{\text {loc }}$ as low as $35 \mathrm{MV} / \mathrm{m}$. The complete energy distribution is shown in Fig. 2(c). While the ponderomotive energy of trapped electrons should scale as $F_{\text {loc }}^{2}$, we observe saturation in the peak electron energy at $\sim 400 \mathrm{eV}$. While the electron energy loss spectra of tungsten nitride show no 

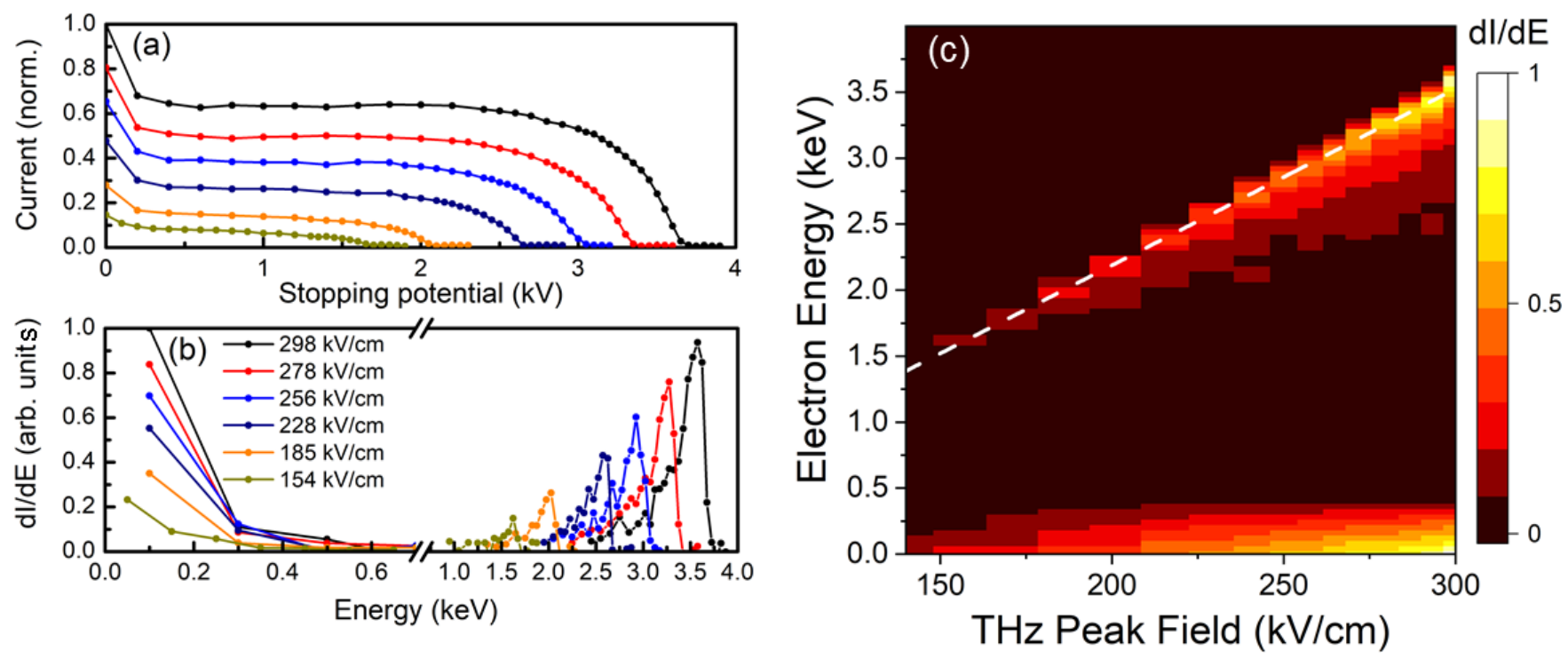

FIG. 2. (a) Electron emission current measured at the Faraday cup detector as a function of the applied retarding potential for THz peak field strength from 154 to $298 \mathrm{kV} / \mathrm{cm}$. Curves are normalized with respect to the $298 \mathrm{kV} / \mathrm{cm}$ maximum current. (b) Energy distributions derived from the raw data, showing low- and high-energy peaks, respectively, which correspond to two qualitatively distinct types of electrons emission. The low-energy tails in the high-energy peak were cut out for clarity. (c) Mapping of the energy distribution as a function of the THz peak field strength.

excitations in the 100-400 eV energy range, an onset of strong electron absorption above $400 \mathrm{eV}$ coincides with the nitrogen $1 s$ excitation [34-36]. We therefore assign the low-energy distribution to electrons inelastically scattered within the nitride, delaying emission and accelerated by the Sommerfeld resonance of the tip. Further evidence for electron energy transfer to surface adsorbates is given later when we examine the nanoscale structure of the tip after long-term exposure. A quantitative analysis will need a more in-depth study of their origin which requires a different experimental setup.

A linear field dependence of the high-energy peak is observed in Fig. 2(c), as expected for ballistic acceleration. The peak energy is given by $U_{\max }=e F_{\mathrm{loc}} l_{\mathrm{F}}$, where $l_{\mathrm{F}}$ is the effective length scale of the field enhancement. The linear fit (white dashed line) with a slope of $13.3 \mathrm{eV} \mathrm{cm} / \mathrm{kV}=e \eta l_{\mathrm{F}}$ directly yields the local field enhancement factor $\eta=3800$ for $l_{\mathrm{F}}=35 \mathrm{~nm}$, approximated as the tip radius. The $F_{\mathrm{loc}}$ in this simple estimate is as high as $115 \mathrm{~V} / \mathrm{nm}$, more than eight times the critical field $F_{\phi}$. The timescales for electrons to leave the field enhancement region are on the order of $5 \mathrm{fs}$, well within the quasistatic emission regime. The corresponding adiabaticity parameter $\delta=l_{\mathrm{F}} m \omega^{2} / e F_{\text {loc }} \approx 0.001$, relating the near-field decay length to the electron quiver amplitude, is also well within the quasistatic limit [16].

\section{Extreme field limit test of Fowler-Norheim theory}

In the quasistatic limit, the Fowler-Nordheim (FN) equations can simulate the emission dynamics of the high-energy distribution. The emission current and final electron energy were calculated using a time-dependent, one-dimensional finite-element simulation taking into account the near-field decay of a hyperboloidal tip $\eta /[1+2 x / R]$, [11,37] with $\eta=$ 1380 and $R=35 \mathrm{~nm}$, the Gaussian $\mathrm{THz}$ field focus, and the $\mathrm{THz}$ pulse waveform in Fig. 1(b). The high-energy electrons rapidly leave the field enhancement region, however still experience the entire $\mathrm{THz}$ waveform before leaving the $\mathrm{THz}$ focal region. Electrons are only slightly slowed down by the subsequent field half cycle before they escape the free-space focal spot. Figure 3(a) shows the local field half cycle and the resultant electron emission rate up to several thousand electrons/fs and occurring in a subcycle burst approximately $200 \mathrm{fs}$ in duration. The onset of such large emission rates occurs when the local field reduces the potential barrier to zero, or $F_{\text {loc }}=F_{\phi}$ (see Fig. 8 in the Appendix). Electrons are chirped in energy according to their near-instantaneous acceleration through the local field in varying parts of the cycle, with the highest energies being emitted in a sub-100-fs duration. At these field strengths, FN theory predicts a much sharper peaked energy distribution than we and others have observed experimentally [10,11], shown in Fig. 3(b) as a black line with a FWHM of $\approx 1 \mathrm{eV}$ on the high-energy peak. To simulate experimental conditions that average over thousands of laser shots, we add statistical fluctuations of the $\mathrm{THz}$ pulse amplitude represented by a truncated Gaussian distribution with a standard deviation of $2 \%$ centered on the measured $\mathrm{THz}$ peak field, a good approximation to our shot-to-shot fluctuations. The calculated energy distribution is obtained by averaging and is shown in Fig. 3(b) (red line), in excellent agreement with the measured high-energy distribution (blue circles). Moreover, the inset shows the single-shot distribution need not match the experimentally observed emission peak energy due to the asymmetric emission process and chirp under the half cycle of the pulse. Thus the single-shot energy distribution is expected to be extremely monochromatic in this high-field regime, with a spectral purity on the order of $10^{-4}$. Thus the THz-driven nanotip is expected to be an extremely bright electron source, potentially useful for single-shot ultrafast electron microscopy and diffraction experiments with sub-100-fs temporal resolution. The addition of space-charge 

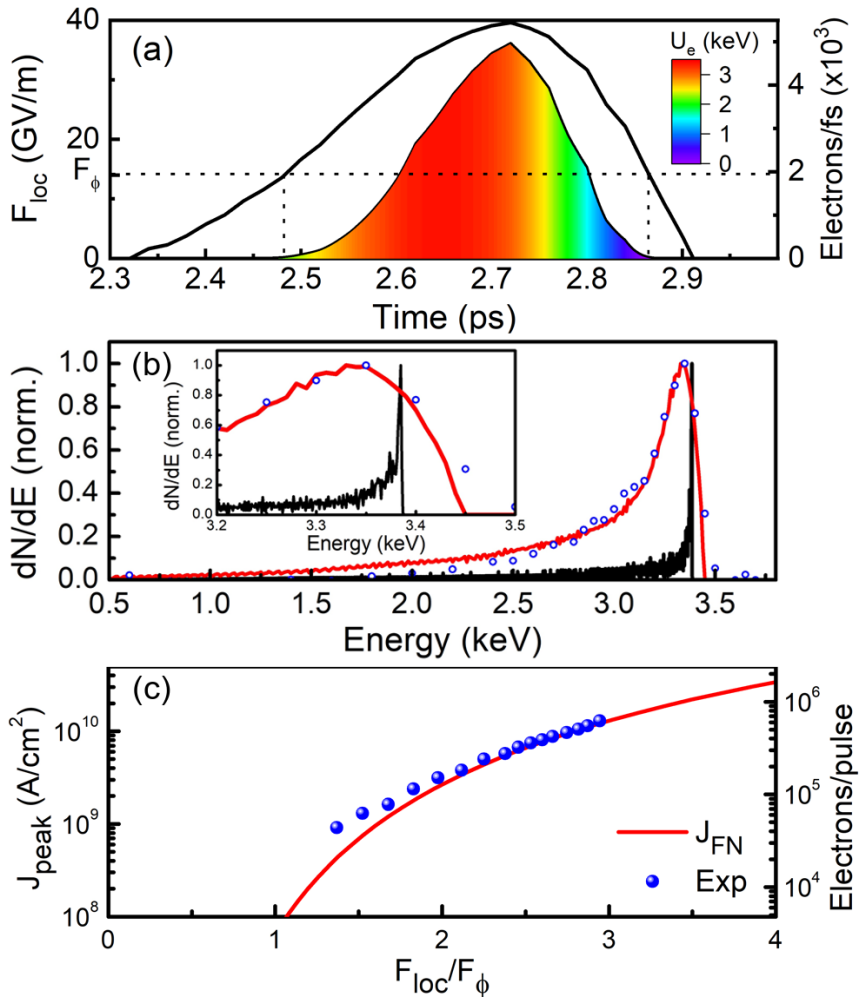

FIG. 3. (a) Local THz pulse electric field $F_{\text {loc }}$ and simulated electron emission rate as described in the text. The chirp of the electron pulse is shown in the color scale. (b) Experimental normalized electron energy distribution (blue circles) at an incident $\mathrm{THz}$ peak electric field of $285 \mathrm{kV} / \mathrm{cm}$. A simulation of the single-shot normalized electron energy distribution (black line) using FowlerNordheim cold-field emission theory and ballistic acceleration of the electrons by the local electric field, as well as with a $2 \%$ Gaussian peak field fluctuation (red line). Inset: Data plotted in the high-energy portion of the energy spectrum. (c) THz peak field dependence of the total high-energy electron per pulse from the nanotip. Low-energy electrons (below $600 \mathrm{eV}$ ) are removed from the electron yield.

effects in the energy calculation lead to a nonlinear relationship of the high-energy peak position and the applied $\mathrm{THz}$ electric field (see Fig. 5 in the Appendix), which we do not observe experimentally.

The peak current density $J_{\text {peak }}$ and bunch charge/pulse under varying $\mathrm{THz}$ peak field is shown in Fig. 3(c). At the maximum fields, $J_{\text {peak }}$ exceeds $10^{10} \mathrm{~A} / \mathrm{cm}^{2}$ with the maximum bunch charge of $10^{6}$ electrons/pulse. Such current densities under dc field conditions would be completely dominated by space-charge effects, governed by the ChildLangmuir law with $J \propto\left(F_{\text {loc }} / F_{\phi}\right)^{3 / 2}$ [38]. The onset of space-charge effects is expected to occur for $F_{\text {loc }} / F_{\phi} \sim 0.5$ for dc field emission [27]. For pulsed field emission from a nanotip, however, space-charge effects should be suppressed under the condition that the pulse duration $(\sim 200 \mathrm{fs})$ is much longer than the electron transit time through $l_{\mathrm{F}}(<5 \mathrm{fs})$. We find that in this extreme strong-coupling regime, space-charge effects are negligible as the emission is well described by a simple one-dimensional Fowler-Nordheim theory with no compensation for local image charges or screening $(v=1)$.

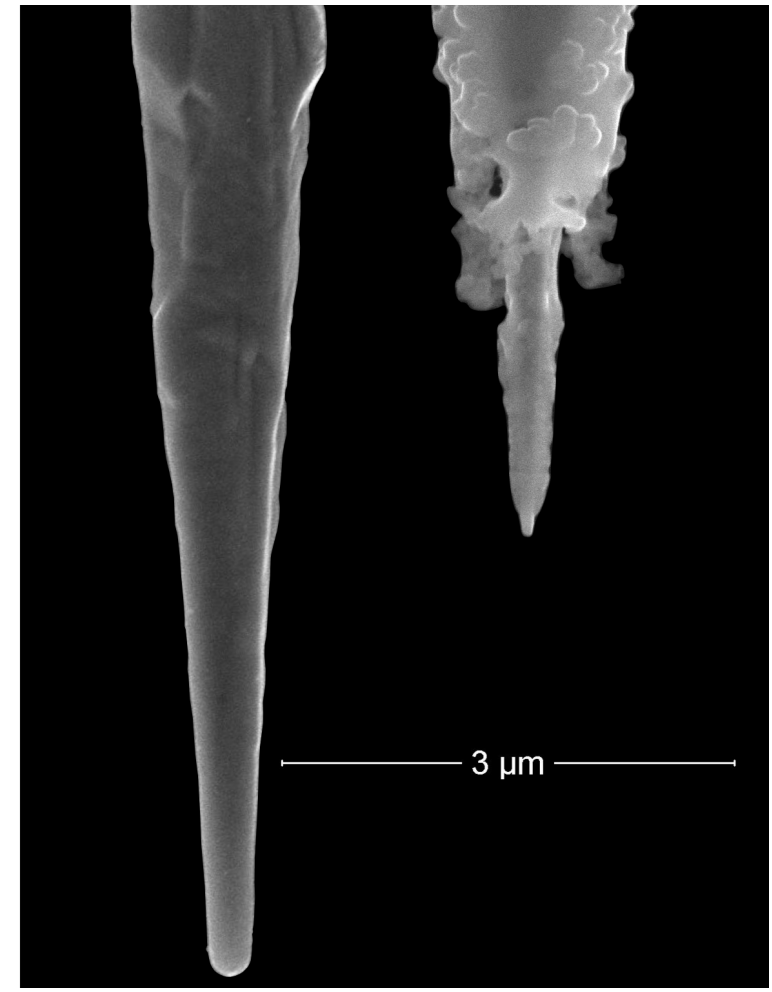

FIG. 4. Scanning electron microscopy image of a tungsten nanotip without exposure (120-nm radius) (left) and with exposure to $\sim 1$ billion of $\mathrm{THz}$ shot (35-nm radius) (right) showing a strong reshaping/sharpening of the apex of the tungsten nanotip improving the electron emission rate and energy.

\section{E. THz field driven nanotip sharpening}

Comparative scanning electron microscope images of a freshly fabricated tungsten nanotip and a tip exposed to $\sim 10^{9}$ high-field $\mathrm{THz}$ pulses are shown in Fig. 4, showing a strong reshaping of the apex of the tip. Over the course of several weeks of experiments, the tip length was reduced by approximately $3 \mu \mathrm{m}$ in length and the tip apex sharpened from a $\sim 120$ to $35 \mathrm{~nm}$ radius. We note that over the course of an experiment in several hours to days, the emission rate was stable. Such field-induced sharpening of a nanotip has been previously observed in field-ion microscopy under applied dc bias voltage, and was used to produce single atom-terminated nanotips [39]. The mechanism for such dc field reshaping was determined to be the formation of tungsten nitride, causing a protrusion that in turn caused a local field enhancement. The local field then exceeded the evaporation threshold for the nitride and the atoms were removed. We therefore tentatively attribute the observed sharpening of the nanotip under intense $\mathrm{THz}$ fields to field evaporation of tungsten nitride on a shot-to-shot basis. However, given the observed low-energy electron distribution, we consider an alternative mechanism could be inelastic scattering of accelerated electrons within the nitride causing the removal of atoms in a similar manner. In this case, electrons transfer energy to regions with tungsten nitride, which are then selectively evaporated in the field, thus sharpening the tip over time on a shot-to-shot basis. 


\section{CONCLUSIONS}

In conclusion, we have demonstrated sub-cycle $\mathrm{THz}-$ driven cold-field electron emission from a tungsten nanotip under extreme local field conditions. Electron bunch charges and peak current densities on the order of $10^{6}$ electrons/pulse and $10^{10} \mathrm{~A} / \mathrm{cm}^{2}$ are quantitatively measured. Energies distribution up to $3.5 \mathrm{keV}$ are observed and are accurately described by Fowler-Nordheim emission with ballistic acceleration in a quasistatic field. Seeing no signature of saturation in the emission current for increasing $F_{\text {loc }}$, combined with the field-induced sharpening of the nanotip and the predicted single-shot spectral purity of $10^{-4}$ indicates that $\mathrm{THz}$-driven nanotip field emission holds promise for ultrabright, ultrafast electron sources.

\section{ACKNOWLEDGMENTS}

D.G.C. and B.J.S. gratefully acknowledge support from FRQNT, NSERC, and the Canada Foundation for Innovation (CFI). We thank Simon L. Lange and Prof. Peter Uhd Jepsen (Technical University of Denmark) for initial discussions and 3D simulations. We also thank Prof. Frank Hegmann (University of Alberta) for useful discussions.

\section{APPENDIX}

\section{Time-dependent finite-element simulations}

Time-dependent finite-element simulations have been performed to simulate the electron emission during the $\mathrm{THz}$ pulse to fit the electron energy distribution using the field enhancement factor as the only fitting parameter. In the acceleration process of the electron, no space charge is considered. The shape and position of the high-energy peak is accurately fit by a $2 \%$ fluctuation in the incoming field and a local electric field of $F_{\text {loc }}=\eta F_{\mathrm{THz}}$ as shown in Fig. 3(b). The high-energy peak position is linear with the incident field as shown in Fig. 2(c). A simple space-charge attenuation was modeled at high electric field using a local field given by

$$
F_{\mathrm{loc}}=\frac{\eta F_{\mathrm{THz}}}{1+\frac{\eta F_{\mathrm{THz}}}{F_{\text {crit }}}} .
$$

Since the high field is attenuated, the field enhancement is increased to reach the same high-energy peak position. The shape of the peak can still be fit while including space charge with similar fluctuations $(2.5 \%)$. However, the position of the high-energy peak is not linear as shown in Fig. 5. It deviates the most from the experimental data at small critical field $\left(F_{\text {crit }}=49 \mathrm{GV} / \mathrm{m}\right)$ and gets closer when the critical field is increased. The best fit corresponded to $F_{\text {crit }}=\infty$ which is without space-charge attenuation. Thus we conclude that space-charge effects are negligible in the calculation of the energy spectrum.

\section{Quiver to subcycle emission regime}

In light-field electron acceleration, two regimes are relevant: the quiver regime and the subcycle regime [10]. The subcycle, quasistatic regime is characterized by a sharp, highenergy peak in the electron energy distribution, tailing to

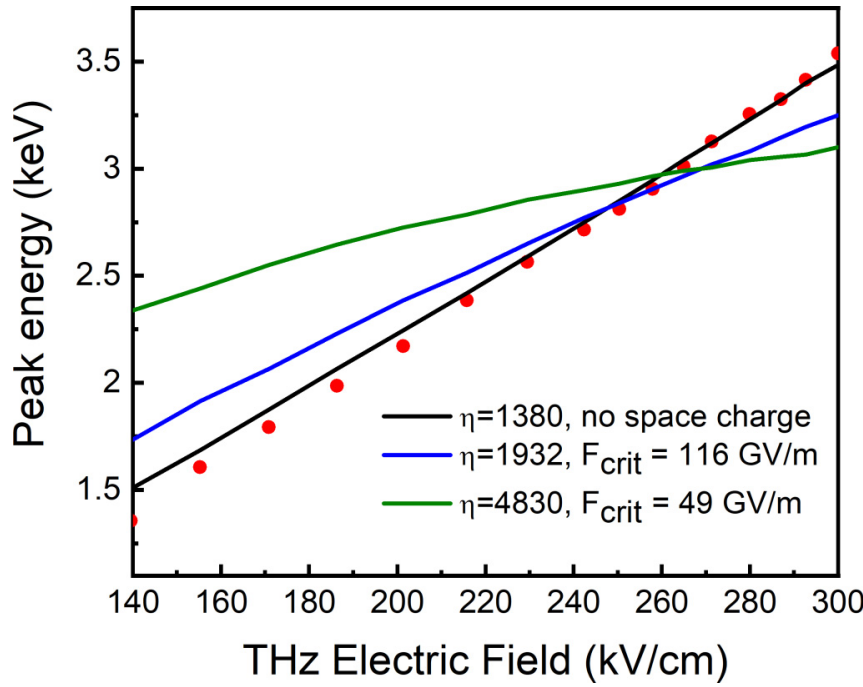

FIG. 5. High-energy peak position of experimental (red dot) and simulation data without (black line) and with space charge (blue and green line) for different critical fields as a function of incident $\mathrm{THz}$ peak electric field.

lower energies. Electrons are ejected at the highest emission rate near the peak of the $\mathrm{THz}$ lightwave, and are accelerated out of the field enhancement region on timescales much shorter than the cycle time. Their final energy is then directly related to the field at the time of their emission. At lower fields, electrons emitted during the $\mathrm{THz}$ peak do not leave the enhancement region fast enough and are slowed down by the next half cycle or even multiple cycles of the THz pulse, leading to a broadened peak. This multicycle interaction is the so-called quiver regime. Simulations of the emission process were performed to find the transition between the two regimes under our experimental conditions. We input our experimentally measured $\mathrm{THz}$ electric field wave forms obtained by free-space electro-optic sampling as our incoming light field. A field enhancement factor of $\eta=1380$ was found to describe the energy distribution with the functional form of the nearfield decay taken to be that of a hyperboloidal tip $\frac{\eta}{1+\frac{2 x}{R}}[11,37]$, for a $R=35 \mathrm{~nm}$ tip radius. We used the zero-temperature Fowler-Nordheim equations (with $v=1$ ) to obtain the electron emission at any given time during the $\mathrm{THz}$ pulse in the quasistatic limit (Keldysh parameter $\gamma_{\mathrm{K}} \ll 1$ ). The energies of the electrons are calculated using a time-dependent finite-element simulation including the $\mathrm{THz}$ pulse Gaussian focus. For our experimental THz peak fields, between 140 and $300 \mathrm{kV} / \mathrm{cm}$, all simulations showed characteristic subcycle emission. Figure 6 shows the normalize energy distribution for $\mathrm{THz}$ peak fields of $10-50 \mathrm{kV} / \mathrm{cm}$. A clear transition between the quiver regime and the subcycle regime is exhibited. The high-energy peak appears around $25 \mathrm{kV} / \mathrm{cm}$ and becomes dominant at $30 \mathrm{kV} / \mathrm{cm}$. Already at $50 \mathrm{kV} / \mathrm{cm}$, the distribution resembles the $285 \mathrm{kV} / \mathrm{cm}$ energy distribution shown in Fig. 3 of the main text. It is important to note that because the pulsed nature of the experiment and the exponential behavior of the field-emission equation, the emission rate per pulse is greatly reduced at lower $\mathrm{THz}$ fields with rates below 1 electron per pulse at $40 \mathrm{kV} / \mathrm{cm}$. We were unable to measure any emission 


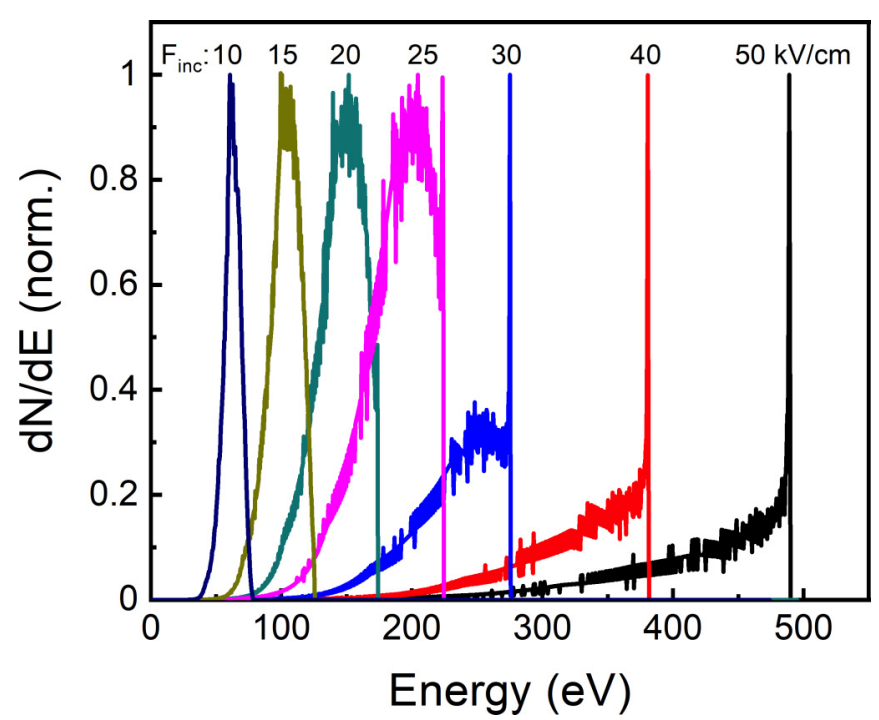

FIG. 6. Time-dependent finite-element simulations of the normalized electron energy distributions for peak $\mathrm{THz}$ electric fields of $10,15,20,25,30,40$, and $50 \mathrm{kV} / \mathrm{cm}$, considering a field enhancement at the tip apex of 1380 .

experimentally with our current setup through the transition regime.

\section{Critical field $\boldsymbol{F}_{\phi}$}

Cold-field emission occurs when a high electric field is applied to a material and bends the potential barrier enough that electrons can start tunneling from an occupied energy level within the material. The Schottky-Nordheim potential is given by the work function $\phi=4.5 \mathrm{eV}$ for tungsten minus the potential of the local electric field and the image potential created by the electrons leaving the material, $V(z)=$ $\phi-e F_{\mathrm{loc}} z-\frac{e}{16 \epsilon_{n} z}$. Fowler-Nordheim theory assumes that the emitted electrons come from the Fermi level. For high enough

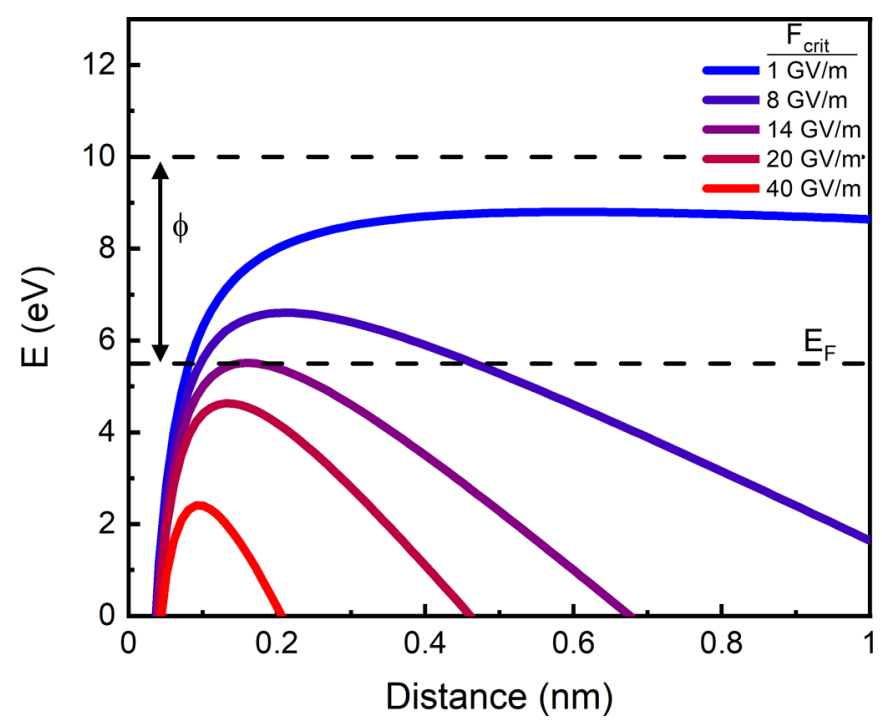

FIG. 7. Schottky-Nordheim potential barrier for electron coldfield emission at different local field strengths.

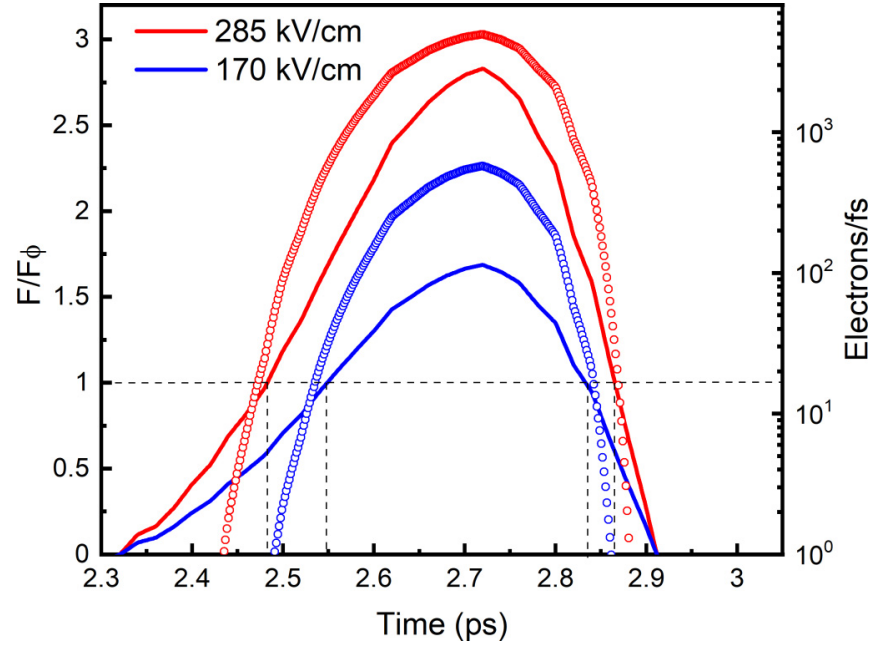

FIG. 8. The normalized local electric field (line) and the electron emission rate (open circles) for a $\mathrm{THz}$ peak field of $285 \mathrm{kV} / \mathrm{cm}$ (red) and $170 \mathrm{kV} / \mathrm{cm}$ (blue).

field values, the height of the potential barrier can be reduced to zero at the critical field $F_{\phi}=4 \pi \epsilon_{0} \phi^{2} / e=14 \mathrm{GV} / \mathrm{m}$, and can even go below the Fermi energy, with $\phi$ given in $\mathrm{eV}$. Figure 7 shows the potential barrier of a tungsten tip $(\phi=$ $4.5 \mathrm{eV}$ ), for a local electric field below, above, and at the critical field. Above the critical field, electrons at the Fermi energy do not have any barrier to tunnel through, making the theory inapplicable. Thus we elected to use a simpler version of the Fowler-Nordheim equation by removing the image term in the potential and consider a simple triangular barrier of height $\phi$ above the Fermi energy. The local electric field used in our experiment exceeds the critical field $F_{\phi}$. Figure 8 shows that the onset of the emission occurs at that critical field for

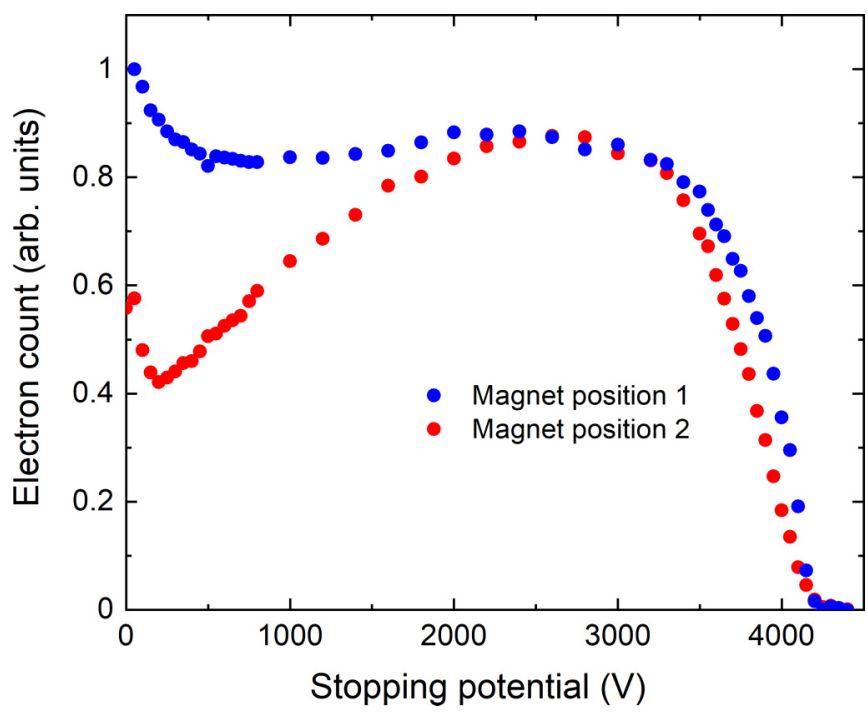

FIG. 9. The normalized electron current as a function of detector grid stopping potential in the presence of a strong magnetic field normal to the electron trajectory supplied by a magnet placed in two separate positions spaced $1 \mathrm{~cm}$ apart. The deflection of the low-energy electrons is apparent as the count rate is considerably lowered for position 2 . 
(a)
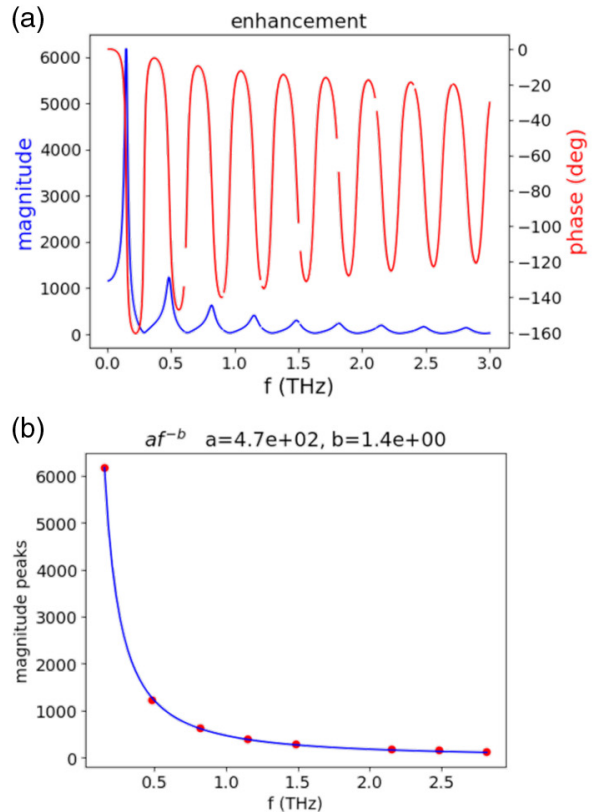

(c)

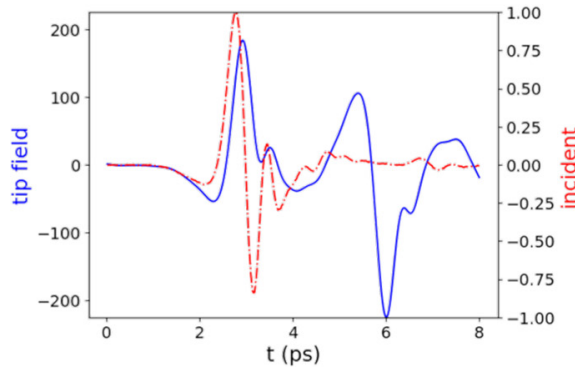

(d)

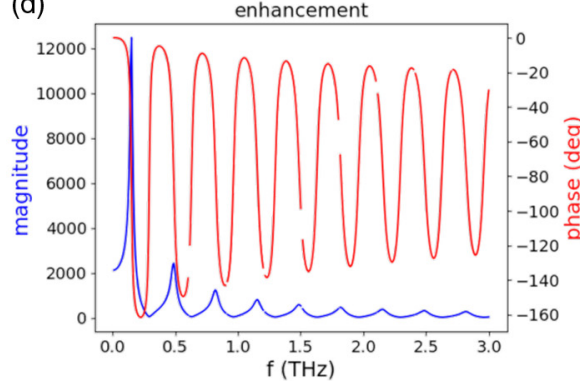

(e)

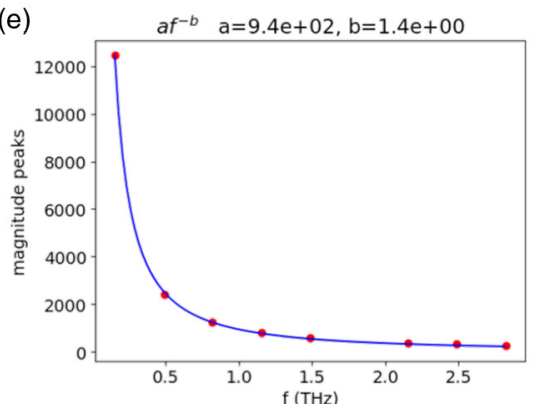

(f)

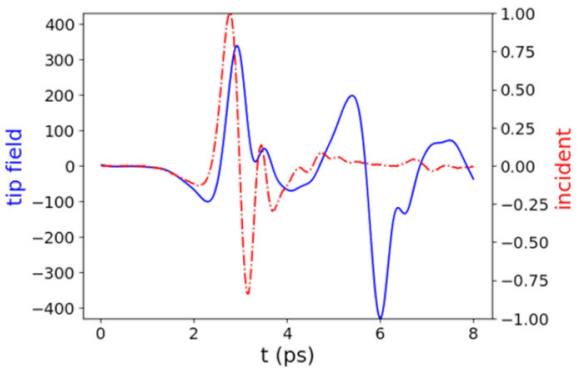

(g)

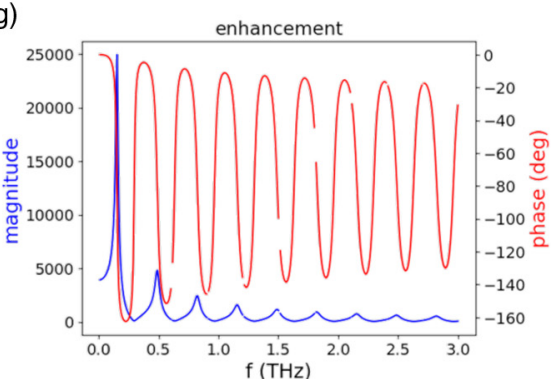

(h)

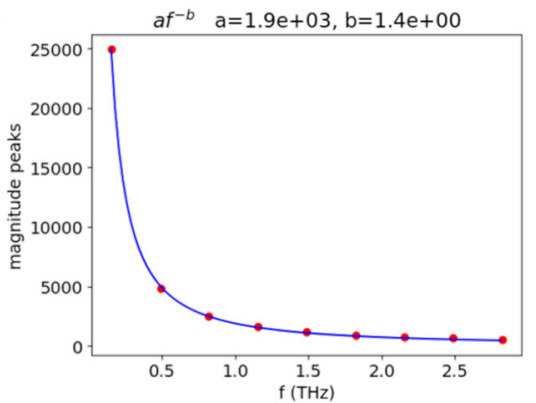

(i)

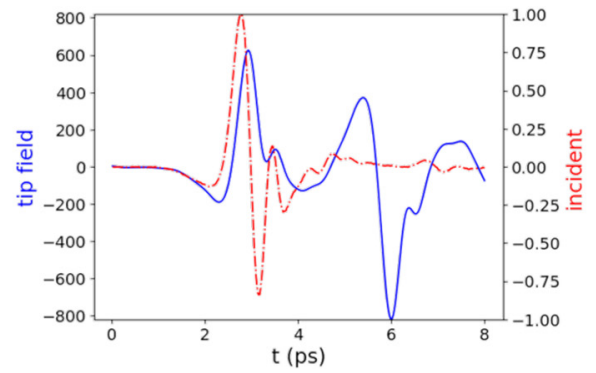

FIG. 10. Three-dimensional finite-difference time-domain (FDTD) simulations calculating (a), (d), (g) the magnitude (blue) and phase (red) of the enhancement factor with respect to the field frequency. Calculations have been made at three different tip radii: (a) $-(\mathrm{c}) R=100 \mathrm{~nm}$, (d)-(f) $R=50 \mathrm{~nm}$, and (g)-(i) $R=25 \mathrm{~nm}$. A nanotip length of $0.9 \mathrm{~mm}$ was used in all calculations. (b), (e), (h) Fit of the decay in the peaks of the enhancement factor magnitude. (c), (f), (i) Comparison of the incident electric field normalized by the experimental THz field (red) and the reconstructed field at the apex of the tip (blue).

both $285-$ and $170-\mathrm{kV} / \mathrm{cm} \mathrm{THz}$ peak electric field. Also, the exponential term in the equation is less important at high fields and the electron pulse duration is reduced at lower fields.

\section{Magnetic field deflection}

A small permanent $\mathrm{Nd}$ disk magnet was used to investigate the point of origin of the detected electrons. Figure 9 shows the detected electron count as a function of stopping potential for two different positions of the magnet along the emission axis outside the vacuum chamber. The orientation of the field was normal to the emission axis. A strong deflection was observed when the magnet was brought in proximity to the beam axis, with pronounced deflection at lower energies. This indicates all detected electrons originate from the $\mathrm{THz}$ illuminated nanotip and are not, for example, due to secondary electron emission at the grid.

\section{3D finite-difference time-domain simulations}

To better understand the temporal structure of the $\mathrm{THz}$ field at the apex of the nanotip, three-dimensional finite-different time-domain calculations were performed modeling the tip as a prolate spheroid. The tip is assumed to be a perfect electrical conductor. Figure 10 shows the results for tip radii of $R=100,50$, and $25 \mathrm{~nm}$, plotting the local field enhancement factor (magnitude and phase), the decay function of the field enhancement, and the temporal structure of the incident and enhanced $\mathrm{THz}$ fields. The length of the tip in these simulations was $0.9 \mathrm{~mm}$, much shorter than the experimental tips $\sim 8-10 \mathrm{~mm}$. This distance corresponds to half the spheroid length and yields interference features in the field enhancement with a free spectral range of $0.33 \mathrm{THz}$ and a roundtrip time of 3 ps. These resonant fields likely will not occur in the experimental geometry. Note the local field experiences a phase lag with regards to the incident field, which is expected to occur in the experiment. Finally, the dispersion of the 
near-field enhancement factor is found to strongly favor the low frequencies, following a power law of the form $\eta=a f^{-b}$ with the $a$ parameter increasing for smaller tip radii and the exponent $b=1.4$ independent of radii.
[1] M. Krüger, C. Lemell, G. Wachter, J. Burgdörfer, and P. Hommelhoff, Attosecond physics phenomena at nanometric tips, J. Phys. B 51, 172001 (2018).

[2] M. Krüger, M. Schenk, M. Förster, and P. Hommelhoff, Attosecond physics in photoemission from a metal nanotip, J. Phys. B 45, 074006 (2012).

[3] R. H. Fowler and L. Nordheim, Electron emission in intense electric fields, Proc. R. Soc. London, Ser. A 119, 173 (1928).

[4] E. L. Murphy and R. H. Good, Thermionic emission, field emission, and the transition region, Phys. Rev. 102, 1464 (1956).

[5] L. V. Keldysh, Ionization in the field of a strong electromagnetic wave, J. Exp. Theor. Phys. 20, 1307 (1965).

[6] T. Rybka, M. Ludwig, M. F. Schmalz, V. Knittel, D. Brida, and A. Leitenstorfer, Sub-cycle optical phase control of nanotunnelling in the single-electron regime, Nat. Photonics 10, 667 (2016).

[7] B. Wolter, M. G. Pullen, M. Baudisch, M. Sclafani, M. Hemmer, A. Senftleben, C. D. Schröter, J. Ullrich, R. Moshammer, and J. Biegert, Strong-Field Physics with Mid-IR Fields, Phys. Rev. X 5, 021034 (2015).

[8] S. S. Wellershoff, J. Hohlfeld, J. Güdde, and E. Matthias, The role of electron-phonon coupling in femtosecond laser damage of metals, Appl. Phys. A 69, S99 (1999).

[9] L. Wimmer, G. Herink, D. R. Solli, S. V. Yalunin, K. E. Echternkamp, and C. Ropers, Terahertz control of nanotip photoemission, Nat. Phys. 10, 432 (2014).

[10] G. Herink, L. Wimmer, and C. Ropers, Field emission at terahertz frequencies: AC-tunneling and ultrafast carrier dynamics, New J. Phys. 16, 123005 (2014).

[11] S. Li and R. R. Jones, High-energy electron emission from metallic nano-tips driven by intense single-cycle terahertz pulses, Nat. Commun. 7, 13405 (2016).

[12] K. E. Echternkamp, G. Herink, S. V. Yalunin, K. Rademann, S. Schafer, and C. Ropers, Strong-field photoemission in nanotip near-fields: From quiver to sub-cycle electron dynamics, Appl. Phys. B 122, 80 (2016).

[13] S. L. Lange, N. K. Noori, T. M. B. Kristensen, K. Steenberg, and P. U. Jepsen, Ultrafast THz-driven electron emission from metal metasurfaces, J. Appl. Phys. 128, 070901 (2020).

[14] W. D. Kilpatrick, Criterion for vacuum sparking designed to include both rf and dc, Rev. Sci. Instrum. 28, 824 (1957).

[15] J. W. Wang and G. A. Loew, RF breakdown studies in copper electron linac structures, in Proceedings of the 1989 Particle Accelerator Conference, Chicago, IL (IEEE, New York, 1989), p. 1137.

[16] G. Herink, D. R. Solli, M. Gulde, and C. Ropers, Field-driven photoemission from nanostructures quenches the quiver motion, Nature (London) 483, 190 (2012).

[17] E. A. Nanni, W. R. Huang, K.-H. Hong, K. Ravi, A. Fallahi, G. Moriena, R. J. Dwayne Miller, and F. X. Kärtner, Terahertzdriven linear electron acceleration, Nat. Commun. 6, 8486 (2015)

[18] C. Ropers, D. R. Solli, C. P. Schulz, C. Lienau, and T. Elsaesser, Localized Multiphoton Emission of Femtosecond
Electron Pulses from Metal Nanotips, Phys. Rev. Lett. 98, 043907 (2007).

[19] E. Quinonez, J. Handali, and B. Barwick, Femtosecond photoelectron point projection microscope, Rev. Sci. Instrum. 84, 103710 (2013).

[20] H. Daoud, K. Floettmann, and R. J. Dwayne Miller, Compression of high-density $0.16 \mathrm{pC}$ electron bunches through high field gradients for ultrafast single shot electron diffraction: The compact RF gun, Struct. Dyn. 4, 044016 (2017).

[21] K.-L. Yeh, M. C. Hoffmann, J. Hebling, and K. A. Nelson, Generation of $10 \mu \mathrm{J}$ ultrashort terahertz pulses by optical rectification, Appl. Phys. Lett. 90, 171121 (2007).

[22] H. Hirori, A. Doi, F. Blanchard, and K. Tanaka, Single-cycle terahertz pulses with amplitudes exceeding $1 \mathrm{MV} / \mathrm{cm}$ generated by optical rectification in $\mathrm{LiNbO}_{3}$, Appl. Phys. Lett. 98, 091106 (2011).

[23] A. S. Lucier, Preparation and characterization of tungsten tips suitable for molecular electronics studies, Ph.D. thesis, McGill University, 2004.

[24] A. Yariv, Quantum Electronics (Wiley, New York, 1975).

[25] B. P. Sinha and R. H. MacPhie, Electromagnetic scattering by prolate spheroids for plane waves with arbitrary polarization and angle of incidence, Radio Sci. 12, 171 (1977).

[26] J. Houard, L. Arnoldi, A. Ayoub, A. Hideur, and A. Vella, Nanotip response to monocycle terahertz pulses, App. Phys. Lett. 117, 151105 (2020).

[27] R. G. Forbes, Description of field emission current/voltage characteristics in terms of scaled barrier field values ( $f$-values), J. Vac. Sci. Technol. B 26, 209 (2008).

[28] R. G. Forbes and J. H. B. Deane, Comparison of approximations for the principal Schottky-Nordheim barrier function $v(f)$, and comments on Fowler-Nordheim plots, J. Vac. Sci. Technol. B 28, C2A33 (2010).

[29] R. Bormann, M. Gulde, A. Weismann, S. V. Yalunin, and C. Ropers, Tip-Enhanced Strong-Field Photoemission, Phys. Rev. Lett. 105, 147601 (2010).

[30] H. Yanagisawa, S. Schnepp, C. Hafner, M. Hengsberger, D. E. Kim, M. F. Kling, A. Landsman, L. Gallmann, and J. Osterwalder, Delayed electron emission in strong-field driven tunneling from a metallic nanotip in the multi-electron regime, Sci. Rep. 6, 35877 (2016).

[31] S. Tanuma, C. J. Powell, and D. R. Penn, Calculation of electron inelastic mean free paths (IMFPs) VII. Reliability of the TPP2M IMFP predictive equation, Surf. Interface Anal. 35, 268 (2003).

[32] K. Wang and D. M. Mittleman, Metal wires for terahertz wave guiding, Nature (London) 432, 376 (2004).

[33] K. Wang and D. M. Mittleman, Guided propagation of terahertz pulses on metal wires, J. Opt. Soc. Am. B 22, 2001 (2005).

[34] G. Soto, W. de la Cruz, F. Castillón, J. Díaz, R. Machorro, and M. Farías, Tungsten nitride films grown via pulsed laser deposition studied in situ by electron spectroscopies, Appl. Surf. Sci. 214, 58 (2003). 
[35] Y.-M. Sun, E. Engbrecht, T. Bolom, C. Cilino, J. Sim, J. White, J. Ekerdt, and K. Pfeifer, Ultra thin tungsten nitride film growth on dielectric surfaces, Thin Solid Films 458, 251 (2004).

[36] B. Wicher, R. Chodun, K. Nowakowska-Langier, M. Trzcinski, L. Skowroński, S. Okrasa, R. Minikayev, M. Naparty, and K. Zdunek, Chemical and structural characterization of tungsten nitride $\left(\mathrm{WN}_{x}\right)$ thin films synthesized via Gas Injection Magnetron Sputtering technique, Vacuum 165, 266 (2019).
[37] M. K. Miller, A. Cerezo, M. Hetherington, and G. D. Smith, Atom Probe Field Ion Microscopy (Clarendon Press, Oxford, UK, 1996).

[38] J. P. Barbour, W. W. Dolan, J. K. Trolan, E. E. Martin, and W. P. Dyke, Space-charge effects in field emission, Phys. Rev. 92, 45 (1953).

[39] M. Rezeq, J. Pitters, and R. Wolkow, Tungsten nanotip fabrication by spatially controlled field-assisted reaction with nitrogen, J. Chem. Phys. 124, 204716 (2006). 